\title{
Perturbative Transport Modeling of Cold-Pulse Dynamics in Alcator C-Mod Ohmic Plasmas
}

\author{
P. Rodriguez-Fernandez, ${ }^{1 *}$ A.E. White, ${ }^{1}$ N.T. Howard, ${ }^{1}$ B.A. Grierson, ${ }^{2}$ X. Yuan, ${ }^{2}$ G.M. Staebler, ${ }^{3}$ J.E. Rice, ${ }^{1}$ \\ C. Angioni, ${ }^{4}$ N.M. Cao, ${ }^{1}$ A.J. Creely, ${ }^{1}$ E. Fable, ${ }^{4}$ M.J. Greenwald, ${ }^{1}$ A.E. Hubbard, ${ }^{1}$ J.W. Hughes, ${ }^{1}$ J.H. Irby, ${ }^{1}$ F. Sciortino ${ }^{1}$ \\ ${ }^{1}$ MIT Plasma Science and Fusion Center, Cambridge, Massachusetts 02139, USA \\ ${ }^{2}$ Princeton Plasma Physics Laboratory, Princeton, New Jersey 08540, USA \\ ${ }^{3}$ General Atomics, P. O. Box 85608, San Diego, California 92186-5608, USA \\ ${ }^{4}$ Max Planck Institut für Plasmaphysik, D-85748 Garching, Germany \\ *Email: pablorf@mit.edu
}

\begin{abstract}
Perturbative transport experiments in magnetically confined plasmas have shown, for more than 20 years, that the injection of cold pulses at the plasma edge can trigger the increase of core temperature. For the first time, predictive simulations with the Trapped Gyro Landau Fluid (TGLF) quasilinear transport model demonstrate that the increase of core temperature in some regimes, and lack thereof in other regimes, can be explained by a change in dominant linear micro-instability. The effect of density and plasma current on the cold pulse are well captured by TGLF, including the relative change in position of the temperature flex point as rational surfaces are shifted. Linear stability analysis of simulated density and current scans reveals a competition between trapped electron and ion temperature gradient modes as the main driver of the core transient response. These results further demonstrate that cold-pulse propagation and associated phenomenology are well explained within the local transport paradigm, without resorting to exotic nonlocal effects.
\end{abstract}

\section{Introduction}

Impurity injections at the edge of magnetically confined plasmas have been widely used for trace impurity transport studies [1, 2], where a small number of foreign neutral particles is deposited at the periphery of the plasma and transported inwards via processes that can be modeled as diffusion and convective particle pinches. An increase in the number of injected particles can cause a sharp drop in edge electron temperature [3], predominantly driven by enhanced line-radiation of partially ionized states that live at the edge of the plasma shortly after the injection [4]. Early experiments in the TEXT tokamak [5] revealed a very fast core electron temperature increase as a result of the edge temperature drop. This seemingly exotic 
effect, characteristic of low collisionality plasmas [6], was readily reproduced in several other tokamaks and helical devices, suggesting that the phenomenology of these effects could, in principle, be explained by robust fundamental mechanisms. In this paper, these phenomena will be referred to as temperature inversions, whereas the lack thereof will be referred to as standard core drop, since the expected response of the plasma core to an edge cold-pulse would be, in principle, a time-delayed temperature decrease.

The time-scale of the onset of the temperature increase and the "reversed-polarity" of this phenomenon led many authors to describe this observation as evidence of nonlocal transport [7]. Here, nonlocal effects refer to phenomena that connect regions of the plasma that are separated by more than a few turbulence correlation lengths [8], and thus allow to decouple the turbulence field from local parameters [9]. However, contemporary models for heat transport in magnetically confined plasmas are based on the assumption that local fluxes can be described in terms of local plasma parameters, such that electromagnetic drift-wave-like turbulence is driven by local gradients and results in cross-field transport. Consequently, the ability to reproduce the fast response of the plasma core to edge cold pulses has been viewed for many years as representing an important milestone for local turbulent transport models [10].

\section{Background}

Local transport models were tested in past studies $[11,12,13]$, revealing that a core electron temperature inversion could be explained by multichannel interactions between electrons and ions via core collisional coupling. These pioneering studies established the basis for this work, but the models at the time were lacking important physics, such as the effect of trapped particles, that led to an underprediction of the magnitude and time-scale of the temperature inversion. These modeling studies also required a strong inwardly-propagating ion heat pulse generated by $T_{i} / T_{e}$ stabilization of turbulence at the periphery of the plasma, whose magnitude was not in agreement with that observed experimentally. Collisional equilibration in plasmas that exhibit temperature inversions is probably not enough to account for the core electron heating, which sometimes is much greater than that of the ion channel. Experimental work [12] also showed that ion temperature would not increase during the electron cold-pulse propagation.

A recent study [14] presented a novel explanation based on a local physics mechanism for the longstanding mystery of "nonlocal" effects in perturbative transport experiments, which had long challenged predictive and postdictive modeling capabilities. It was proposed that turbulence stabilization due to flattening of density gradients after cold-pulse injections in plasmas with dominance of trapped-electron- 
mode (TEM) turbulence was the cause of the core electron temperature increase. Likewise, turbulence destabilization arising from an increase of ion temperature gradients in plasmas with dominance of IonTemperature-Gradient (ITG) turbulence could make the temperature inversions disappear.

Even though the model could explain the observations within experimental constraints, there is still a number of experimental properties of cold pulses that remained unexplored:

- Experiments in Tore Supra [15] and Alcator C-Mod [6, 16] showed that core temperature inversions can happen at high-density by raising the plasma current.

- Work in Alcator C-Mod [16] revealed that temperature inversions can exist regardless of the direction of the core intrinsic rotation, and pointed out that the magnitude of the temperature inversions linearly decreased with line-averaged density, instead of experiencing an abrupt transition.

- Experiments in RTP $[17,18,19]$ found that the electron temperature profile flex point was linked to the motion of rational surfaces, an observation that was later confirmed in ASDEX Upgrade [12] and Alcator C-Mod [6].

- Work on TFTR [8] pointed to the ratio between electron-ion collisional power and electron heat flux as an important metric for the appearance of temperature inversions, which seemed to occur in plasmas when electrons and ions are thermally decoupled. Such correlation of the core response to the ratio between the collisional electron-ion power transfer to electron heat conduction was also confirmed in subsequent experiments in RTP [17].

Any model that aims at explaining temperature inversions must also reproduce the full phenomenology observed for more than twenty years in tokamak plasmas. The aim of this paper is to address these issues by means of new integrated modeling simulations that incorporate poloidal field diffusion and a triggering model for sawtooth crashes, as well as an extension of the parameter ranges that were used in previous work [14].

\section{Methodology}

As described in Ref. [14], the model consists of a set of implicit transport equations, solved using the PT_SOLVER numerical scheme [20] integrated with the TRANSP power balance code [21, 22], and coldpulse injections are modeled by artificially perturbing radiation and impurity density profiles. In this work, we perform a numerical experiment based on the low-density condition reported in [14]. Around this condition, scans of electron density and plasma current are performed, to resolve whether the model 
captures the observed trends of temperature inversions with changes in electron density, plasma current and safety factor profile. Following the literature $[6,16]$, plasma current effects will be tested for $I_{p}=$ 0.55 MA (low), 0.8 MA (medium) and 1.1 MA (high). Likewise, volume-averaged density will cover a range of $\left\langle n_{e}\right\rangle \approx 0.5-1.5 \cdot 10^{20} \mathrm{~m}^{-3}$, which is close to the observed cold-pulse transition for Alcator CMod plasmas.

To accomplish this, poloidal field diffusion is self-consistently solved, along with the introduction of sawteeth crashes to prevent current peaking and unrealistic on-axis temperature profiles. The MHD equilibrium is solved using the TEQ library [23], with fixed plasma boundary. This is motivated by the fact that changes in plasma position or magnetic flux surfaces due to impurity injections were demonstrated experimentally to be too small to affect the core temperature response or measurement [5, 24].

The time dependent evolution of the highly non-linear and stiff equations for electron and ion temperatures are solved using Newton iterations over 200 equally spaced radial zones and adaptive time-steps. Neoclassical transport is estimated using the Chang-Hinton analytic model [25], and turbulent transport fluxes are calculated using the Trapped Gyro-Landau Fluid (TGLF) quasilinear model [26, 27], with a saturation rule that accounts for cross-scale coupling physics (TGLF-SAT1) [28, 29]. Temperature profiles are solved from a boundary condition at $\rho_{N}=0.9$ (square root of the normalized toroidal flux) up to the magnetic axis. No transport enhancement factors are included, in order to test the actual transport physics within the model. The poloidal wavenumber grid $\left(k_{\theta}\right)$ normalized at each radius by the ion acoustic gyroradius $\left(\rho_{s}\right)$ for the turbulent transport simulations is chosen from $k_{\theta} \rho_{s} \approx 0.1$ up to $k_{\theta} \rho_{s} \approx$ 24.0. Beyond the boundary condition at $\rho_{N}=0.9$, measured plasma profiles are kept constant during the scans. We have made sure that this choice does not compromise convergence of the transport solver in the outer radii when plasma parameters vary.

To limit the complexity of the simulations and ease the interpretation of the results, the ion effective charge, $Z_{e f f}$, is assumed unchanged during the density and current ramps, as well as the impurity fractional content (except at the cold-pulse injection times, when impurities are injected and travel inwards, as was done in [14]). Cold pulses are introduced by artificial changes to the radiation and impurity density profiles, as described in [14]. These perturbations are the same for all cold pulses in this numerical experiment, an assumption made here to simplify the interpretation of the phenomena. As the cold pulse propagates, total plasma current and applied magnetic field are kept constant in time. 
During the density scans, density gradient inverse scale lengths, $a / L_{n}$, are also kept constant, and intrinsic rotation is assumed co-current and also unchanged during cold-pulse injections and parameter scans. The authors are aware that, during such parameter ranges, steady-state experimental plasma parameters such as $Z_{\text {eff }}$ or $a / L_{n}$, among others, may change [6,30], thus affecting the background turbulence and confinement. However, here we keep them constant in order to discern whether temperature inversions can be explained solely by changes in dominant micro-instabilities, which will be carefully studied. In terms of the intrinsic rotation, past work [16] showed no changes in the core response during rotation reversals, and suggested that momentum transport may not play a role in the phenomenology of temperature inversions. Recent work in J-TEXT [31] has showed that plasma rotation can change as a consequence of the cold-pulse injection, but it is likely a consequence of the change in dominant microinstability and core turbulence state as the cold pulse travels inwards, rather than a cause of the temperature inversion.

\section{Density Scan}

The most commonly observed feature of the temperature inversion effect since its discovery [5] is its disappearance at high densities. Experimentally, it was proposed [6] that the temperature inversion effect takes place in plasmas with dominance of collisionless trapped electron mode turbulence, and that disappears at higher densities, where ion temperature gradient turbulence becomes important. This conjecture was later confirmed by integrated modeling of Alcator C-Mod plasmas [14], which showed that turbulent transport can be suppressed by the arrival of a density pulse in low-collisionality TEMdominated regimes, while destabilization by ion temperature gradient takes place in ITG plasmas at higher collisionality. This observation was consistent with the confinement saturation paradigm, since it has long been considered that the transition from linear Ohmic confinement (LOC) to saturated Ohmic confinement (SOC) regimes happens concomitant with the destabilization of ITG at higher densities [30]. 
As we describe next, Fig. 1 displays the linear response of the core temperature increase with density. Injections are introduced every $50 \mathrm{~ms}$ during a linear density ramp with constant plasma current. The magnitude of the core temperature inversions (Fig. 1b) following edge temperature drops (Fig. 1c) decreases and the inversion appears later in time as density is increased. Concurrently, electron-ion collisional power becomes closer to the electron heat conduction power (Fig. 1d), indicating that the plasma becomes more thermally coupled, and TEM as the dominant linear micro-instability gets weaker (Fig. 1e). At low densities, the electron conducted power is quickly reduced following the laser blow-off (LBO) injection, a consequence of the stabilization of TEM modes when they are the primary electron heat exhaust mechanism. The electron conducted power starts to increase

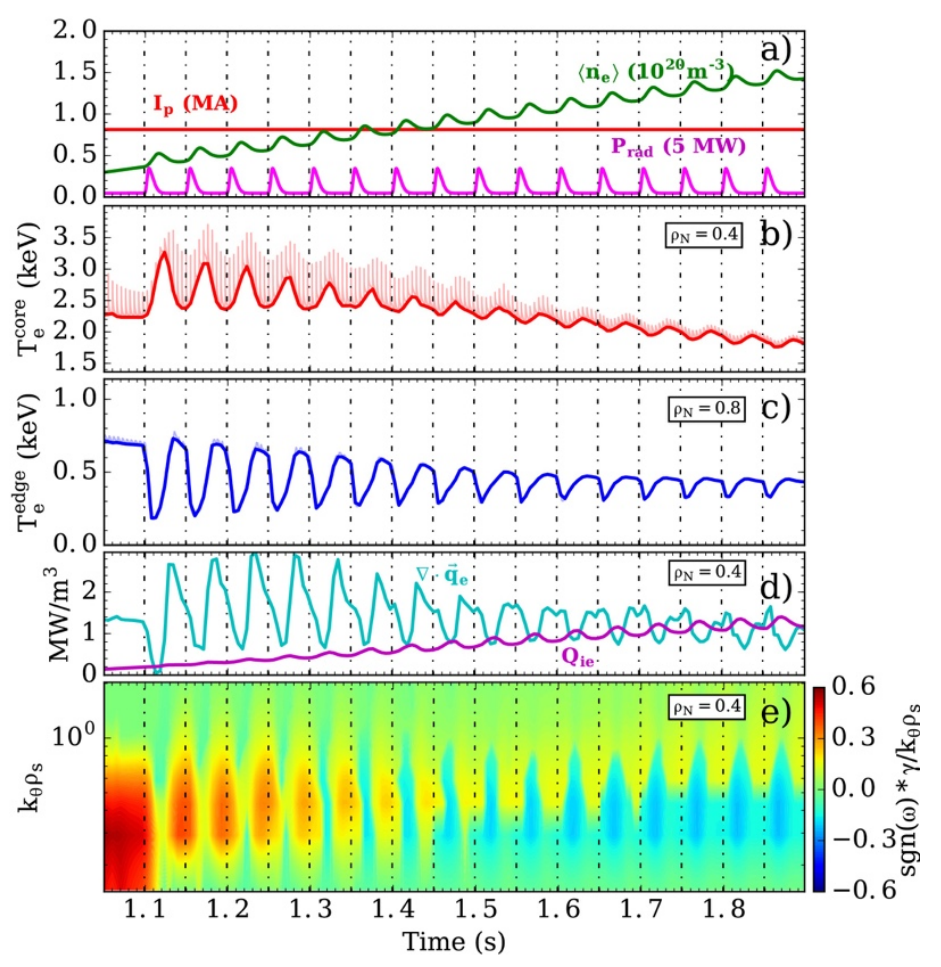

Figure 1. Simulation of density scan. (a) Plasma current, volumeaveraged density and total radiated power. (b) Electron temperature trace at $\rho_{N}=0.4$. (c) Electron temperature trace at $\rho_{N}=0.8$. (d) Electron-ion collisional power and electron conducted power at $\rho_{N}=$ 0.4. (d) Low-k linear growth rates spectrum at $\rho_{N}=0.4$, with sign representing direction of propagation (blue: ion diamagnetic direction; red: electron diamagnetic direction). Vertical dashed lines represent cold-pulse injection times. Spectrum, temperatures and power densities have been down-sampled to before-sawtooth-crash times.

following the injection at around $t=1.4 s$, and consequently the core electron temperature starts exhibiting a temperature drop. The increase in conducted power (and thus decrease in core temperature) gets larger as density increases. Interestingly, the core electron temperature gets above the steady-state value before relaxing. We believe this could be a consequence of the simplified shape of the density pulse, which does not properly capture the relaxation of the density profile. Future work with an experimentally constrained density pulse and self-consistent particle transport prediction will help understand this behavior.

The linear response of the core temperature with density and the continuous (instead of abrupt) transition has been observed experimentally [16]. Our simulation captures this behavior, which is fundamentally different from other abrupt transport phenomena, such as the core intrinsic rotation reversal or hollowing [32]. For some years, a causal relationship between rotation reversal density and the disappearance of the 
temperature inversion effect was suggested, but here it is shown that the temperature inversions can happen regardless of the direction of the toroidal rotation (in all cases, co-current rotation is assumed), and that the disappearance is not abrupt. Instead, a weakening of the core temperature inversion is clearly observed. While here we provide evidence that the phenomenology of the heat transport channel is not directly affected by the direction of plasma rotation and its gradients, it is likely that intrinsic rotation is affected by the turbulence state of the plasma core [33, 32].

\section{Current Scan}

For sawtoothing plasmas $\left(q_{0} \approx 1.0\right)$ at high plasma current (low $\left.q_{95}\right)$, the safety factor profile becomes flatter at the plasma core and the locations of rational surfaces generally move outwards. Cold-pulse experiments in several machines (RTP [17, 18, 19], ASDEX-U [12] and Alcator C-Mod [6]) revealed that the electron temperature "flex point" (the radial location where the electron temperature remains the same before and during the cold-pulse propagation) was linked to the safety factor profile, and that the location moves along with the rational surfaces. In order to test this experimental property of the cold-pulse paradigm, we ran a simulation at three currents $\left(I_{p}=\right.$ $0.55 M A, 0.8 M A, 1.1 M A)$ and low enough density $\left(\left\langle n_{e}\right\rangle \approx 0.5 \cdot 10^{20} \mathrm{~m}^{-3}\right)$ so that inversions happen at the three currents, as depicted in Fig. 2a and Fig. $2 b$.

Fig. 2c plots the normalized linear growth rate of low-k modes $\left(k_{\theta} \rho_{s}=0.6\right)$ only for electrondirection modes when they are dominant. One can see how, by raising the current, electron modes become more unstable, covering larger portions of the plasma. The consequence of this behavior is that, for a given location in the plasma, TEMs are more unstable as we raise the current, and the inversion happens in outer positions because electron heat flux becomes more sensitive to the TEM drives at those outer locations. Such change of microturbulence in

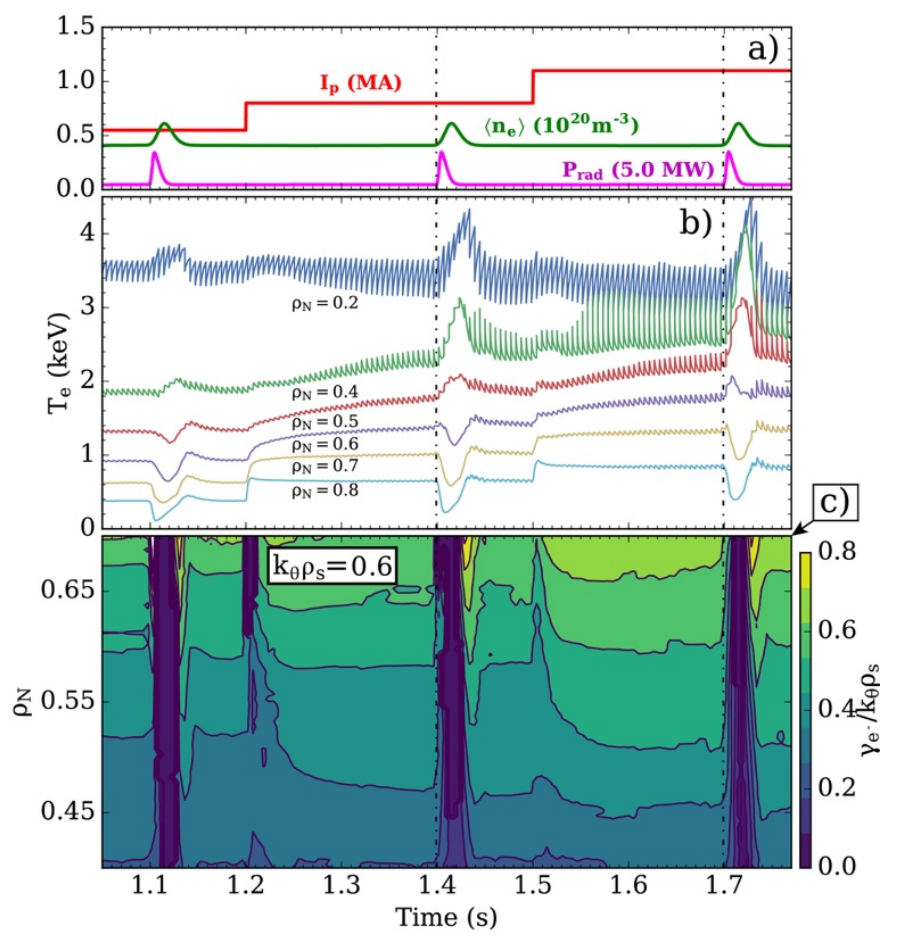

Figure 2. Simulation of current scan. (a) Plasma current, volumeaveraged density and total radiated power. (b) Electron temperature traces at several radial locations. (c) Linear growth rates at $k_{\theta} \rho_{s}=0.6$ in the electron diamagnetic direction. Vertical dashed lines represent cold-pulse injection times. Spectrum has been down-sampled to before-sawtooth-crash times. 
the outer portions of the plasma volume is likely related to the reduction of collisionality that happens when the electron temperature increases (as more Ohmic power is provided) at constant density. Lower collisionality leads to less de-trapping and consequently more TEM activity. The lower magnetic shear associated with the increase in plasma current in sawtoothing plasmas (i.e. with $q_{0} \approx 1.0$ ) also contributes to higher transport levels.

Fig. 3a shows the electron temperature profiles before and after $(10 \mathrm{~ms})$ cold-pulse injections, and the temperature flex point is also plotted explicitly. Figure $3 \mathrm{~b}$ shows that the qualitative trends are captured by the simulation: the temperature flex point moves outwards with increasing plasma current, following the motion of the rational surfaces. However, the temperature flex point in the simulation is systematically over-predicted, which could be related to the over-prediction of steadystate core electron temperature and consequent underprediction of collisionality [14]. Such core temperature over-prediction could be due to two main reasons. On the one hand, effective ion charge and main ion dilution, among other steady-state plasma parameters, are not well constrained from experiment and can have a significant effect on the TEM instability. On the other hand, the over-prediction of core electron temperature could be related to the under-prediction of quasilinear weights in TGLF. To compensate this under-prediction, the transport solver increases $a / L_{T e}$ until the fluxes that result from the power balance
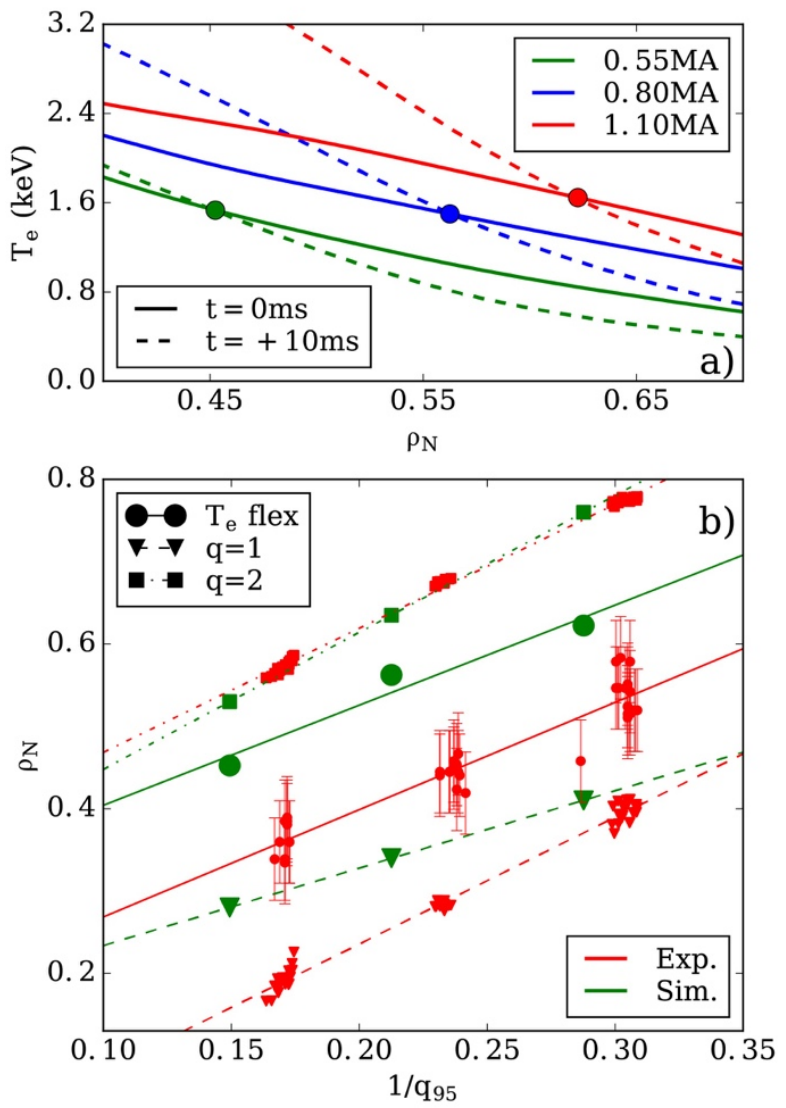

Figure 3. (a) Simulated core temperature profile (solid) before and (dashed) 10ms after the cold-pulse injection for the three plasma currents: 0.55MA (green), 0.8MA (blue) and 1.1MA (red). (b) Simulated and experimental temperature flex point and location of $q=1$ and $q=2$ for different values of $q 95$. Experimental flex points adapted from [Rice NF 2013].

calculation and the fluxes from the transport model are matched. Given that the saturation rule is constructed from linear physics, the flux compensation results in a situation with more unstable linear modes than in experiment. As the integration of "over-predicted" gradients leads to lower collisionality (higher core temperatures for constant density), trapped electron modes will be particularly over-excited in relation to ion temperature gradient modes. In any case, if one takes into account that there might be a 
threshold in the ratio of TEM to ITG turbulence levels to have temperature inversions (as the data and the simulations suggest), the stronger TEM turbulence resulting from the over-prediction of the steady-state profiles in TGLF could be leading to temperature inversions in outer radii and with larger magnitude than in experiment.

\section{Combined effect of density and plasma current}

The disappearance of temperature inversions at high-density has been well-documented in several experimental studies [34 and references therein]. However, work in Alcator C-Mod [16] showed experimental evidence of core temperature inversions at very high densities when the plasma current is raised. Such modification of the "transition density" by plasma current had also been observed in Tore Supra [15].

Fig. $4 \mathrm{a}$ and Fig. $4 \mathrm{~b}$ demonstrate that the simulation captures the full phenomenology: (1) temperature inversion at low-density, (2) disappearance following a density increase, (3) re-appearance after increasing the plasma current. Fig. 1c shows that the LBO injection that does not exhibit a temperature inversion (injection \#2) features a collisional equilibration power roughly equal to the electron heat conduction power, whereas the latter is much larger for injections \#1 and \#3. This is consistent with past work $[8,17]$ suggesting that temperature inversions correlate with the ratio of collisional electronion power transfer to electron heat conduction, i.e. temperature inversions take place in thermally decoupled (or weakly coupled) plasmas. Fig. $4 d$ shows the linear growth rates spectrum at $\rho_{N}=0.4$, whose sign indicates the direction of propagation. The

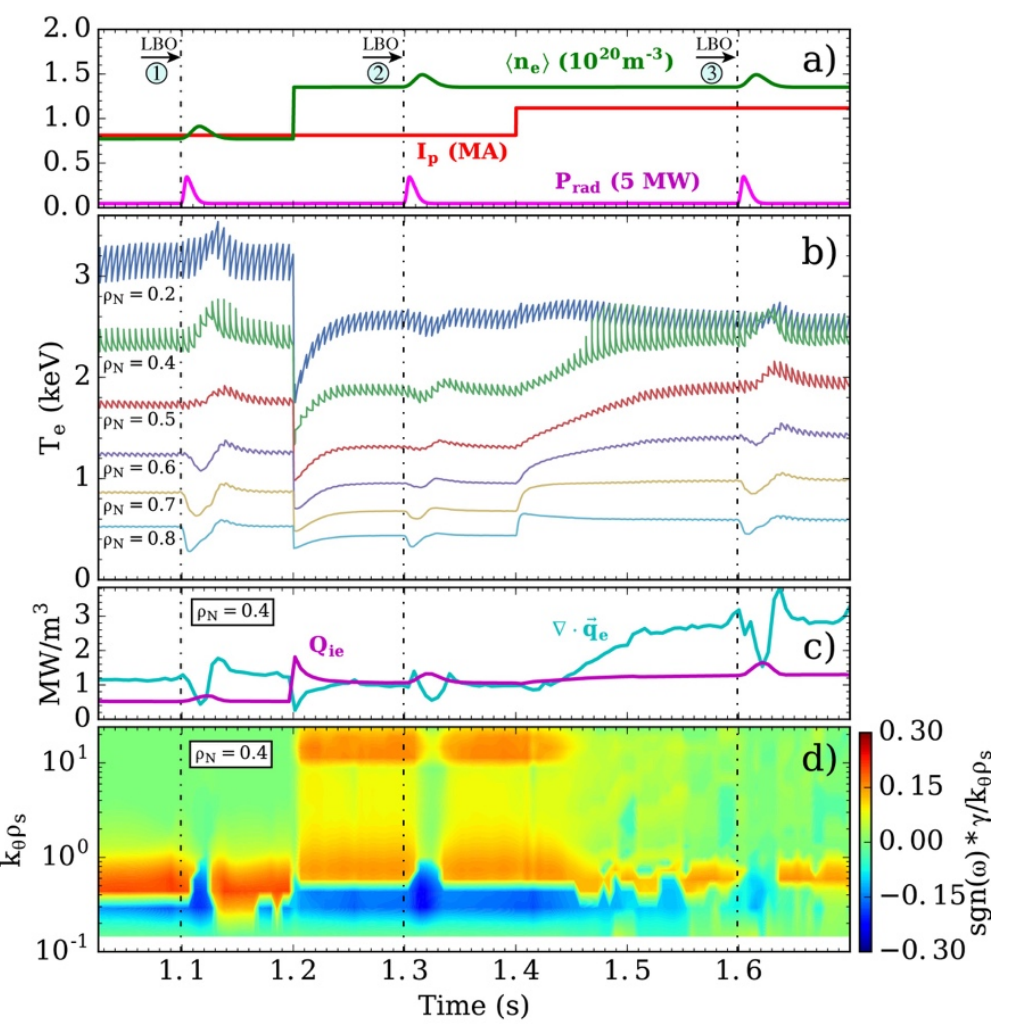

Figure 4. Simulation of combined density and current scan. (a) Plasma current, volume-averaged density and total radiated power. (b) Electron temperature traces at several radial locations. (c) Electron-ion collisional power and electron conducted power at $\rho_{N}=0.4$. (d) Linear growth rates spectrum at $\rho_{N}=0.4$, with sign representing direction of propagation (blue: ion diamagnetic direction; red: electron diamagnetic direction). Vertical dashed lines represent cold-pulse injection times. Spectrum and power densities have been down-sampled to beforesawtooth-crash times. 
linear stability spectrum is dominated at long wavelengths $\left(k_{\theta} \rho_{s} \lesssim 1.0\right)$ by electron modes for those conditions where the temperature inversion takes place. The plasma at the time of injection $\# 2$, which exhibits a standard core drop, is dominated by ITG modes at long wavelengths and Electron Temperature Gradient (ETG) modes at short wavelengths. Even though TEMs are indeed stabilized by the flattening of density gradients and increase in collisionality (Fig. 4d), the loss of TEM dominance in exhausting electron heat flux prevents the prompt reduction of electron transport, as it will be discussed in greater detail in the next sections.

\section{Analysis of the inversion condition from linear characteristics}

Simulations of cold-pulse propagation suggest that the existence of the temperature inversion effect is somehow related to the dominant linear micro-instability in the plasma core. In order to quantify this, a metric is constructed based on the relative strength of the estimated mixing length transport of TEM v.s. ITG. For every cold pulse simulated in this work, the TGLF gyrofluid model is used to calculate the dominant linear modes for each sign of the frequency (electron and ion diagmagnetic directions), and a dominance metric is formulated here as $\eta_{T E M / I T G}=D_{T E M} / D_{I T G}$, where $D_{T E M}=\max \left[\gamma_{T E M} / k_{T E M}{ }^{2}\right]$ and $D_{I T G}=\max \left[\gamma_{I T G} / k_{I T G}^{2}\right]$.

Because cold pulses are introduced during continuous density ramps (i.e. with changes in background temperature) and a small temperature drop may happen before the temperature increase (as seen in Figure 1), a metric for the strength of the temperature inversion must also be constructed. In this study we use $\Delta T_{e}^{\text {core }}=\frac{1}{t f-t 0} \int_{t 0}^{t f}\left(T_{e}-T_{e, 0}\right) d t$, where $T_{e, 0}$ is the linear interpolation of the temperature without a cold pulse, $t_{0}$ is the cold-pulse injection time, and $t_{f}$ is the time after the effect disappears. This means that, if the plasma exhibits a core temperature increase larger than the initial core temperature drop, the plasma is considered to exhibit a temperature inversion. Fig. 5 depicts the relationship between these two metrics at $\rho_{N}=0.4$

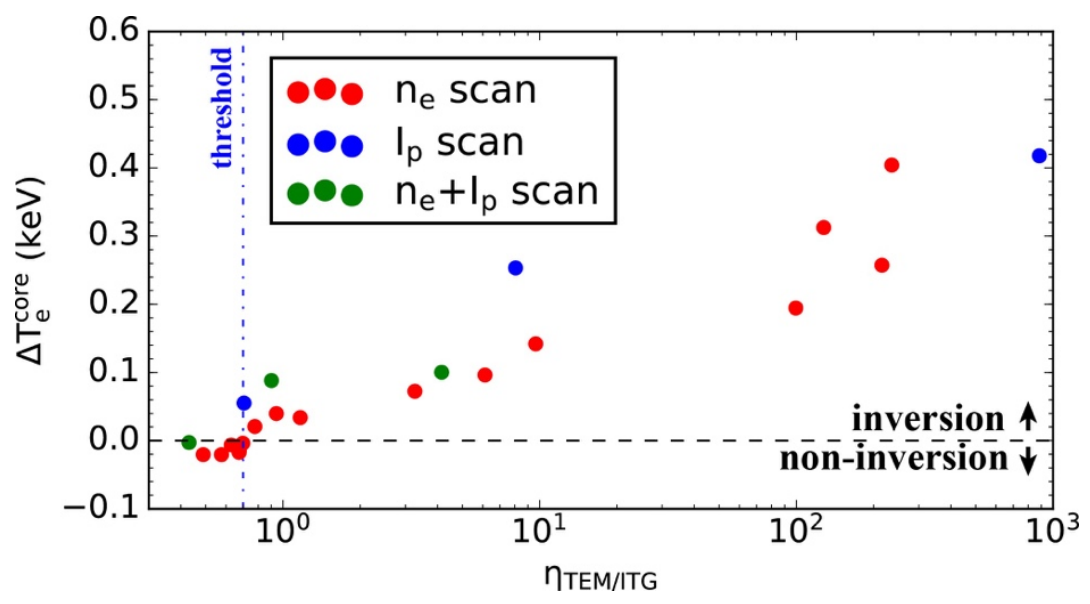

Figure 5. Change in core electron temperature as a function of TEM V.s. ITG dominance for all cold pulses simulated in this paper: cold pulses from (red) Figure 1, (blue) Figure 2, and (green) Figure 4. 
for all plasmas in this paper. It is clearly observed that temperature inversions get stronger as the TEMs are more dominant in relation to the ITG modes. Using this metric, temperature inversions disappear when the TEM transport (estimated with $D_{T E M}$ ) becomes lower than $\sim 70 \%$ of ITG (estimated with $D_{I T G}$ ).

\section{Analysis of driving terms of TEM v.s. ITG dominated regimes}

In the previous section, the existence of temperature inversions or lack thereof is shown to be correlated with the dominant micro-instability at the plasma core. However, the details of how the turbulence state can give rise to such different core temperature dynamics remained unclear. In order to address this, detailed linear stability analysis at a time before and after the cold-pulse injection is performed for the three injections in Fig. 4.
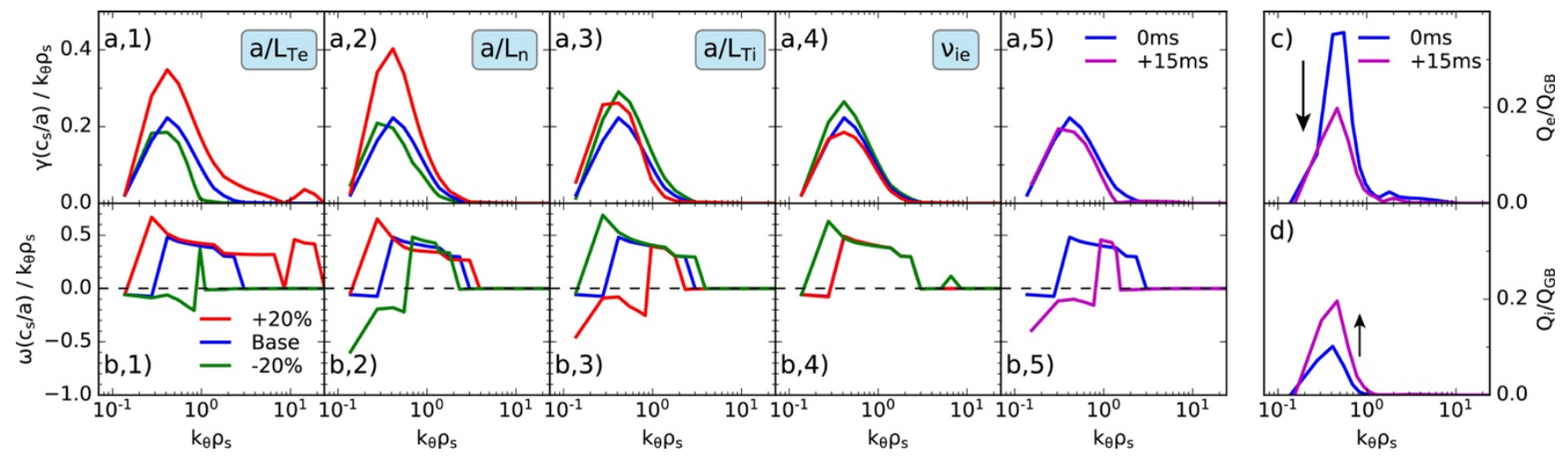

Figure 6. Analysis of Injection \#1 from Fig. 4. (a) Linear growth rates and (b) frequencies of the most unstable mode at $\rho_{N}=0.4$. Scans for 4 main driving terms are shown in red (-20\%) and green (+20\%), with respect to the baseline (blue). The spectrum after the coldpulse arrival (magenta) is also plotted. (c) Electron and (d) ion heat flux spectra before and after the cold-pulse arrival.

As shown in Fig. 6, this analysis confirms that TEMs are the dominant micro-instability in the core of the low-density, medium-current plasma (injection \#1 in Fig. 4). As shown in the subset of Figures 6a and 6b, linear stability is most sensitive to changes in both electron temperature and density gradients, as indicated by a larger increase in the growth rate with changes in those drives (red curves). Ion temperature gradient, on the other hand, is shown to destabilize modes in the ion direction but stabilize modes in the electron direction, which is a known feature of TEMs [35, 36, 37]. After the injection (15ms), TEMs are stabilized and the dominant micro-instability is in the ion diamagnetic drift direction at low-k, as shown in Fig. 6a,5) and Fig. 6b,5). As TEMs are more efficient at exhausting electron heat flux than ITG modes at low collisionality, electron heat flux is reduced (Fig. 6c), leading to the increase of core electron temperature. 
Next, we study injection \#2 in Fig. 4 (highdensity medium-current), where it is observed that the spectrum becomes ITG-dominated at long wavelengths and ETG-dominated at short wavelengths by raising the density, leaving a small window in between for existence of TEMs. Because of this, after the pulse arrives ITG modes are destabilized whereas TEM/ETG modes are stabilized (Fig. 7a and Fig. 7b), but not enough to compensate the increase in long wavelength electron transport due to the ITG enhancement. In this high-density condition,
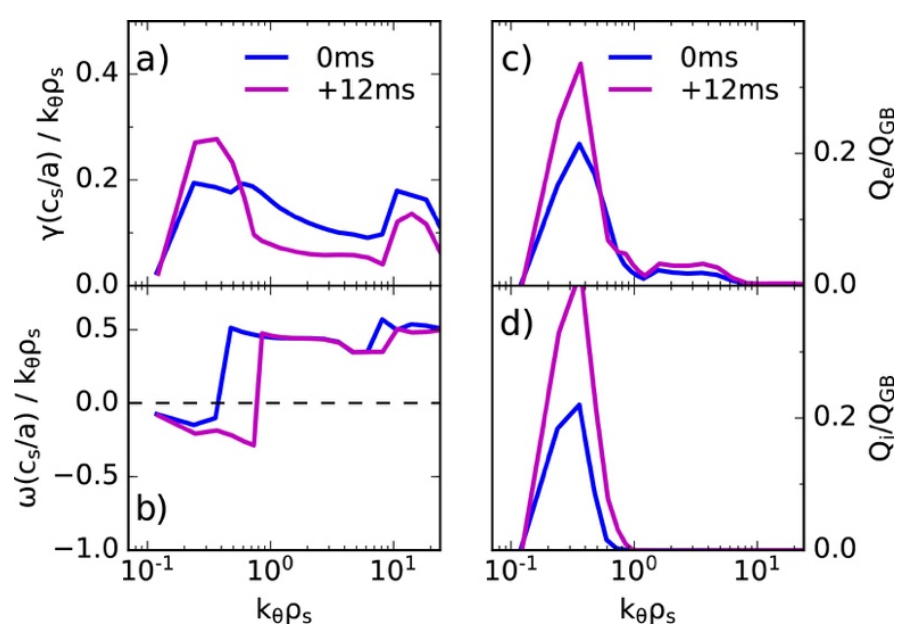

Figure 7. Analysis of injection \#2 from Fig. 4. (a) Linear growth rates, (b) mode frequencies, (c) electron and (d) ion heat flux spectra (blue) before and (magenta) after the cold-pulse arrival.

electron transport is dominantly driven at low-k by ITG modes, which become more unstable and drive more turbulence (hence, higher electron transport as well), as shown in Fig. 7c.

Lastly, by raising the plasma current (injection \#3 in Fig. 4), transport is dominated by low-k TEMs, as shown in Fig. 8a and Fig. 8b. The linear spectrum is now mostly affected by density gradients, which suppress electron modes after the cold-pulse arrival. In this case, the electron heat flux spectrum shifts to longer wavelengths, but the total electron heat flux
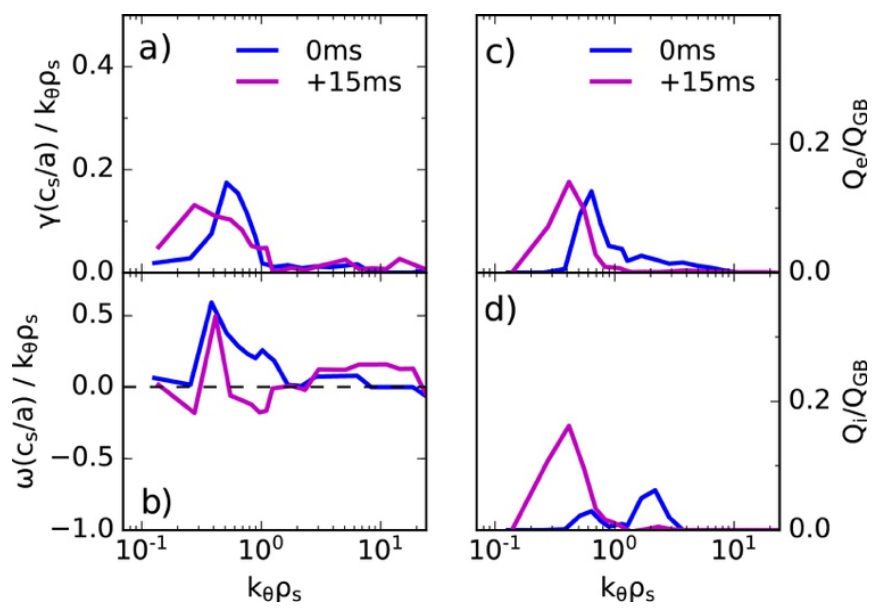

Figure 8. Analysis of injection \#3 from Fig. 4. (a) Linear growth rates, (b) mode frequencies, (c) electron and (d) ion heat flux spectra (blue) before and (magenta) after the cold-pulse arrival. decreases.

From the linear stability analysis of Fig. 7, one can see that changes in the ion temperature gradient are the cause of the core electron temperature drop in the high-density cases, since ITG modes dominate the linear spectrum at long wavelengths. Contrary to the density evolution, which is manually imposed in these simulations, ion temperature dynamics are a consequence of changes in turbulent transport. From Fig. 6d, Fig. 7d and Fig. 8d, we can see that ion heat flux is enhanced in all cases (even in those cases that exhibit an electron heat flux reduction). This increase in ion transport is related to the reduction of impurity 
density gradient, which leads to the destabilization of ITG [35], amplified in some cases by the selfconsistent increase in $a / L_{T i}$.

\section{Conclusions}

This study presents a set of simulations that further confirm that local turbulent transport models can reproduce the phenomenology of cold-pulse propagation in tokamak plasmas. Specifically, it is demonstrated that the well-documented current and density dependencies of cold pulses can be recovered using TRANSP simulations based on the TGLF-SAT1 turbulent transport model. Parametric studies and linear analyses of the dominant micro-instabilities present in the plasma core reveal that the appearance of the temperature inversion effect is strongly correlated with the strength of trapped electron modes, which are more efficient at exhausting electron heat flux than ion temperature gradient modes at low collisionality. The study presented here makes use of the TGLF gyrofluid model, which was developed to include both passing and trapped particles dynamics [26]. Trapped particle modes are indeed shown here to be critical to explain the behavior at low collisionality. Also, our modeling results reveal that ion heat pulses are not needed to explain the electron core temperature increase, consistent with experimental observations in other machines [12]. Further work is needed to investigate the conditions that lead to the increase in ion temperature, observed in some experiments [34].

Cold-pulse experiments have been extensively described in the literature, and many different features have been reported. This paper does not aim at discussing all characteristics of cold-pulse dynamics, but provides confidence that our modeling assumptions are appropriate to reproduce all experimental trends that have so far been discussed. Future work should address whether the model can capture the behavior of cold-pulse propagation with application of different heating schemes. In the work presented here, only

Ohmic discharges were analyzed. It is found experimentally that temperature inversions are enhanced by predominantly electron heating schemes such as electron cyclotron heating (ASDEX-U [12], HL-2A [38]) and Lower Hybrid (Tore Supra [39]), and weakened in ion heating scenarios with neutral beam injections (TFTR [40], JET [13]). These observations are also consistent with the idea of dominance of electron versus ion micro-instabilities at the plasma core, and with the correlation of the magnitude of the inversion with the ratio of electron to ion heat flux. Nevertheless, specific simulations to prove these ideas need to be performed. 
The difficulty of drift-wave-type turbulence driven by local gradients to explain cold-pulse behavior had been considered one of the outstanding flaws of modern transport models based on local gyrokinetics [7]. This work represents a validation exercise for the model presented in [14], which is shown here to reproduce satisfactorily all experimental trends that have been tested so far. Reproducing such complex turbulent transport dynamics with the TGLF model is remarkable, and gives us confidence that the fundamental assumptions of local reduced models alike are sufficient to predict transport and confinement in magnetic-confinement fusion devices.

\section{Acknowledgements}

The authors appreciate insightful discussions with Dr. Mantica, Prof. Gentle and Dr. Citrin on the dynamics of cold-pulse propagation in tokamak plasmas. We thank the Alcator C-Mod team for their excellent work on the original experiments and the TRANSP team for their support with the intensive runs. Data analysis was performed using the OMFIT framework [41, 42]. This material is based upon work supported by the U.S. Department of Energy, Office of Science, Office of Fusion Energy Sciences, using Alcator C-Mod, a DOE Office of Science user facility, under Award No. DE-FC02-99ER54512. P.R.F. was also supported by U.S. Department of Energy Award No. DE- SC0014264 and a La Caixa Fellowship.

\section{References}

[1] N.T. Howard et al Review of Scientific Instruments 82, 033512 (2011); https://doi.org/10.1063/1.3565448

[2] J.E. Rice et al Nucl. Fusion 55033014 (2015); https://doi.org/10.1088/0029-5515/55/3/033014

[3] M.W. Kissick et al 1994 Nucl. Fusion 34349 (1994); https://doi.org/10.1088/0029-5515/34/3/I03

[4] T. Sunn Pedersen et al Review of Scientific Instruments 70, 586 (1999); https://doi.org/10.1063/1.1149487

[5] K.W. Gentle et al Phys. Rev. Lett. 74, 3620 (1995); https://doi.org/10.1103/PhysRevLett.74.3620

[6] J.E. Rice et al Nucl. Fusion 53033004 (2013); https://doi.org/10.1088/0029-5515/53/3/033004

[7] K. Ida et al Nucl. Fusion 55013022 (2015); https://doi.org/10.1088/0029-5515/55/1/013022

[8] J.D. Callen et al Plasma Phys. Control. Fusion 39, B173 (1997); https://doi.org/10.1088/0741-3335/39/12B/014

[9] F. Hariri et al Physics of Plasmas 23, 052512 (2016); https://doi.org/10.1063/1.4951023

[10] E.J. Doyle (Chair Transport Physics) et al Nucl. Fusion 47 S18 (2007); https://doi.org/10.1088/0029-5515/47/6/S02

[11] J.E. Kinsey et al Physics of Plasmas 5, 3974 (1998); https://doi.org/10.1063/1.873117

[12] F. Ryter et al Nucl. Fusion 401917 (2000); https://doi.org/10.1088/0029-5515/40/11/311

[13] P Mantica et al Plasma Phys. Control. Fusion 442185 (2002); https://doi.org/10.1088/0741-3335/44/10/308

[14] P. Rodriguez-Fernandez et al Phys. Rev. Lett. 120, 075001 (2018); https://doi.org/10.1103/PhysRevLett.120.075001 
[15] X.L. Zou et al Plasma Phys. Control. Fusion 421067 (2000); https://doi.org/10.1088/0741-3335/42/10/305

[16] P. Rodriguez-Fernandez et al Nucl. Fusion 57074001 (2017); https://doi.org/10.1088/1741-4326/aa6e89

[17] P. Galli et al 1999 Nucl. Fusion 391355 (1999); https://doi.org/10.1088/0029-5515/39/10/301

[18] P. Mantica et al Phys. Rev. Lett. 82, 5048 (1999); https://doi.org/10.1103/PhysRevLett.82.5048

[19] G.M.D. Hogeweij et al Plasma Phys. Control. Fusion 421137 (2000); https://doi.org/10.1088/0741-3335/42/10/310

[20] X. Yuan et al APS Division of Plasma Physics (2013); http://adsabs.harvard.edu/abs/2013APS..DPPJP8119Y

[21] F. Poli et al TRANSP. Computer Sofware (2018); https://doi.org/10.11578/dc.20180627.4

[22] R. Hawryluk, Proceedings of Phys. Plasmas Close to Thermonucl. Cond. (Elsevier, NY, 1979) Vol. 1, p. 19.

[23] L.L. LoDestro et al Physics of Plasmas 1, 90 (1994); https://doi.org/10.1063/1.870464

[24] M.W. Kissick et al Nucl. Fusion 361691 (1996); https://doi.org/10.1088/0029-5515/36/12/I09

[25] C.S. Chang and F.K. Hinton The Physics of Fluids 25, 1493 (1982); https://doi.org/10.1063/1.863934

[26] G.M. Staebler et al Physics of Plasmas 12, 102508 (2005); https://doi.org/10.1063/1.2044587

[27] G.M. Staebler et al Physics of Plasmas 14, 055909 (2007); https://doi.org/10.1063/1.2436852

[28] G.M. Staebler et al Physics of Plasmas 23, 062518 (2016); https://doi.org/10.1063/1.4954905

[29] G.M. Staebler et al Nucl. Fusion 57066046 (2017); https://doi.org/10.1088/1741-4326/aa6bee

[30] I. Erofeev et al Nucl. Fusion 57126067 (2017); https://doi.org/10.1088/1741-4326/aa8e32

[31] Y. Shi et al Nucl. Fusion 58044002 (2018); https://oi.org/10.1088/1741-4326/aaaa9b

[32] Y. Camenen et al Plasma Phys. Control. Fusion 59034001 (2017); https://doi.org/10.1088/1361-6587/aa543a

[33] Y. Camenen et al Nucl. Fusion 51073039 (2011); https://doi.org/10.1088/0029-5515/51/7/073039

[34] C. Gao et al Nucl. Fusion 54083025 (2014); https://doi.org/10.1088/0029-5515/54/8/083025

[35] H. Du et al Physics of Plasmas 21, 052101 (2014); https://doi.org/10.1063/1.4875342

[36] N. Bonanomi et al Nucl. Fusion 58, 026028 (2018); https://doi.org/10.1088/1741-4326/aa9e7c

[37] J.Y. Jim et al Physics of Plasmas 24, 072501 (2017); https://doi.org/10.1063/1.4990071

[38] H.-J. Sun et al Plasma Phys. Control. Fusion 52045003 (2010); https://doi.org/10.1088/0741-3335/52/4/045003

[40] M. Kissick et al Nucl. Fusion 38821 (1998) https://doi.org/10.1088/0029-5515/38/6/304

[41] B.A. Grierson et al Fusion Technol. 74:1-2, 101-115 (2018); https://doi.org/10.1080/15361055.2017.1398585

[42] O. Meneghini et al Physics of Plasmas 23, 042507 (2016); https://doi.org/10.1063/1.4947204 